\title{
POWER-TYPE QUASIMINIMIZERS
}

\author{
Anders Björn and Jana Björn \\ Linköping University, Department of Mathematics \\ SE-581 83 Linköping, Sweden; anbjo@mai.liu.se \\ Linköping University, Department of Mathematics \\ SE-581 83 Linköping, Sweden; jabjo@mai.liu.se
}

\begin{abstract}
In this paper we examine the quasiminimizing properties of radial power-type functions $u(x)=|x|^{\alpha}$ in $\mathbf{R}^{n}$. We find the optimal quasiminimizing constant whenever $u$ is a quasiminimizer of the $p$-Dirichlet integral, $p \neq n$, and similar results when $u$ is a quasisub- and quasisuperminimizer. We also obtain similar results for $\log$-powers when $p=n$.
\end{abstract}

\section{Introduction}

Let $1<p<\infty$ and let $\Omega \subset \mathbf{R}^{n}$ be a nonempty open set. A function $u \in W_{\text {loc }}^{1, p}(\Omega)$ is a $Q$-quasiminimizer, $Q \geq 1$, in $\Omega$ if

$$
\int_{\varphi \neq 0}|\nabla u|^{p} d x \leq Q \int_{\varphi \neq 0}|\nabla(u+\varphi)|^{p} d x
$$

for all $\varphi \in W_{0}^{1, p}(\Omega)$. Quasiminimizers were introduced by Giaquinta and Giusti [11], [12] as a tool for a unified treatment of variational integrals, elliptic equations and quasiregular mappings on $\mathbf{R}^{n}$. They realized that De Giorgi's method could be extended to quasiminimizers, obtaining, in particular, local Hölder continuity. DiBenedetto and Trudinger [10] proved the Harnack inequality for quasiminimizers, as well as weak Harnack inequalities for quasisub- and quasisuperminimizers. We recall that a function $u \in W_{\text {loc }}^{1, p}(\Omega)$ is a quasisub(super)minimizer if (1.1) holds for all nonpositive (nonnegative) $\varphi \in W_{0}^{1, p}(\Omega)$.

After the papers by Giaquinta-Giusti [11], [12] and DiBenedetto-Trudinger [10], Ziemer [25] gave a Wiener-type criterion sufficient for boundary regularity for quasiminimizers. Tolksdorf [22] obtained a Caccioppoli inequality and a convexity result for quasiminimizers. The results in [10], [11], [12] and [25] were extended to metric spaces by Kinnunen-Shanmugalingam [16] and J. Björn [8] in the beginning of this century, see also A. Björn-Marola [6]. Soon afterwards, Kinnunen-Martio [15] showed that quasiminimizers have an interesting potential theory, in particular they introduced quasisuperharmonic functions, which are related to quasisuperminimizers in a similar way as superharmonic functions are related to supersolutions, see Definition 2.1.

In this paper we study radial quasiminimizers of power-type. Let $\mathbf{B}=B(0,1)$ denote the unit ball in $\mathbf{R}^{n}$. The following is one of our main results.

doi:10.5186/aasfm.2011.3619

2010 Mathematics Subject Classification: Primary 49J20; Secondary 31C45, 35J20.

Key words: Doubling measure, nonlinear, $p$-harmonic, Poincaré inequality, potential theory, quasiminimizer, quasisubharmonic, quasisubminimizer, quasisuperharmonic, quasisuperminimizer. 
Theorem 1.1. Let $1<p<n, \alpha \leq \beta=(p-n) /(p-1)$ and $u(x)=|x|^{\alpha}$. Then $u$ is a $Q$-quasiminimizer in $\mathbf{B} \backslash\{0\}$ and a $Q$-quasisuperharmonic function in $\mathbf{B}$, where

$$
Q=\left(\frac{\alpha}{\beta}\right)^{p} \frac{p \beta-p+n}{p \alpha-p+n}
$$

is the best quasiminimizer constant in both cases.

We also obtain similar results for $p=n$ and $p>n$, thus including the onedimensional case $n=1$, see Theorem 6.1.

So far, there have been very few concrete examples of quasiminimizers for which the best quasiminimizer constant is known. There are of course a few, but not very many, explicit examples of $p$-harmonic functions, i.e. with $Q=1$. In the onedimensional case there are a couple of examples with optimal quasiminimizer constant in Judin [14], Martio [18] and Uppman [24]. As far as we know there are no earlier examples of quasiminimizers with known optimal quasiminimizer constant $Q>1$ in higher dimensions.

Most of the theory for quasiminimizers so far has been extending various results known for $p$-harmonic functions. On the other hand, our examples show that some results are not extendable and the class of quasiminimizers behaves in a way that was not expected.

One of the consequences of Theorem 1.1 is that the best exponent in the weak Harnack inequality for $Q$-quasisuperminimizers must depend on $Q$, and tends to 0 , as $Q \rightarrow \infty$. The same is true for the best exponent of local integrability for $Q$-quasisuperharmonic functions. It also shows that some of the "classical" Caccioppoli type inequalities for superminimizers cannot be true for quasisuperminimizers with exponents independent of the quasiminimizing constant $Q$. See Björn-BjörnMarola [5] for a full discussion of the consequences of Theorem 1.1 that have so far been obtained.

Our examples are also examples of local $(1+\varepsilon)$-quasiminimizers which are not quasiminimizers, showing that being a quasiminimizer is not a local property. We show that this is not surprising and that there are plenty of such examples. As far as we know there is only one explicit example in the literature in this direction, due to Judin [14].

The function $u(x)=|x|^{\beta}$, with $\beta$ and $p$ as in Theorem 1.1, is (up to a constant multiple) the fundamental solution of the $p$-Laplace operator $\Delta_{p}$, i.e. the solution of the equation $\Delta_{p} u=\delta$, where $\delta$ is the Dirac measure at 0 , and is probably the most important superharmonic function. We believe that the quasisuperharmonic functions $u(x)=|x|^{\alpha}$ provided by Theorem 1.1 will turn out to be important in the further studies of quasiminimizers.

The one-dimensional theory of quasiminimizers was already considered in Giaquinta-Giusti [11], and has since been further developed in Martio-Sbordone [21], Judin [14], Martio [18] and Uppman [24]. Most aspects of the higher-dimensional theory fit just as well in metric spaces, and this theory, in particular concerning boundary regularity, has recently been developed further in a series of papers by Martio [17]-[19], A. Björn-Martio [7], A. Björn [1]-[4] and J. Björn [9].

Compared with the theory of $p$-harmonic functions we have no differential equation for quasiminimizers, only the variational inequality can be used. There is also no comparison principle nor uniqueness for the Dirichlet problem. The following result 
was recently obtained by Martio [19], Theorem 4.1. It shows that quasiminimizers are much more flexible under perturbations than solutions of differential equations, which can be useful in applications and in particular shows that results obtained for quasiminimizers are very robust.

Theorem 1.2. Let $u$ be a $Q$-quasiminimizer in $\Omega$ and $f \in W_{\text {loc }}^{1, p}(\Omega)$ be such that $|\nabla f| \leq c|\nabla u|$ a.e. in $\Omega$, where $0<c<Q^{-1 / p}$. Then $u+f$ is a $Q^{\prime}$-quasiminimizer in $\Omega^{\prime}$, where $Q^{\prime}=(1+c)^{p} /\left(Q^{-1 / p}-c\right)^{p}$.

The outline of the paper is as follows: In Section 2 we discuss the basic theory of quasiminimizers and take a first look at radial power-type functions. In Section 3 we determine exactly when powers (and log-powers in the case $p=n$ ) are suband superminimizers and sub- and superharmonic. These results are well known but we need to record them for later use. In Sections 4-7, we study exactly when powers are quasiminimizers, quasisub- and quasisuperminimizers and quasisub- and quasisuperharmonic and determine the best $Q$ 's in all cases. In Section 7 we also obtain similar results for log-powers in the case $p=n$. In Section 8 we provide examples of local quasiminimizers and show that being a quasiminimizer is not a local property.

Acknowledgement. The authors were both supported by the Swedish Research Council, and belong to the European Science Foundation Networking Programme Harmonic and Complex Analysis and Applications and to the Scandinavian Research Network Analysis and Application.

\section{Quasi(super)minimizers}

Our definition of quasiminimizers (and quasisub- and quasisuperminimizers) is one of several equivalent possibilities, see Proposition 3.2 in A. Björn [1]. In fact it is enough to test (1.1) with (all, nonpositive and nonnegative, respectively) $\varphi \in \operatorname{Lip}_{c}(\Omega)$, where $\operatorname{Lip}_{c}(\Omega)$ denotes the set of all Lipschitz functions with compact support in $\Omega$. Note also that a function is a $Q$-quasiminimizer in $\Omega$ if and only if it is both a $Q$-quasisubminimizer and a $Q$-quasisuperminimizer in $\Omega$.

By Giaquinta-Giusti [12], Theorem 4.2, a $Q$-quasiminimizer can be modified on a set of measure zero so that it becomes locally Hölder continuous in $\Omega$. A $Q$-quasiharmonic function is a continuous $Q$-quasiminimizer.

Kinnunen-Martio [15], Theorem 5.3, showed that if $u$ is a $Q$-quasisuperminimizer in $\Omega$, then its lower semicontinuous regularization $u^{*}(x):=\operatorname{ess}_{\lim \inf _{y \rightarrow x} u(y) \text { is }}$ also a $Q$-quasisuperminimizer in $\Omega$ belonging to the same equivalence class as $u$ in $W_{\text {loc }}^{1, p}(\Omega)$. Furthermore, $u^{*}$ is $Q$-quasisuperharmonic in $\Omega$. For our purposes we make the following definition.

Definition 2.1. A function $u: \Omega \rightarrow(-\infty, \infty]$ is $Q$-quasisuperharmonic in $\Omega$ if $u$ is not identically $\infty$ in any component of $\Omega, u$ is lower semicontinuously regularized, and $\min \{u, k\}$ is a $Q$-quasisuperminimizer in $\Omega$ for every $k \in \mathbf{R}$.

A function $u: \Omega \rightarrow[-\infty, \infty)$ is $Q$-quasisubharmonic in $\Omega$ if $-u$ is $Q$-quasisuperharmonic in $\Omega$.

This definition is equivalent to Definition 7.1 in Kinnunen-Martio [15], see Theorem 7.10 in [15]. (Note that there is a misprint in Definition 7.1 in [15] - the functions $v_{i}$ are assumed to be $Q$-quasisuperminimizers.) 
A function is $p$-harmonic if it is 1-quasiharmonic, it is sub(super)harmonic if it is 1-quasisub(super)harmonic, and it is a (sub/super)minimizer if it is a 1-quasi(sub/ super)minimizer.

We will need the following removability result. Here $C_{p}$ is the Sobolev capacity, see Heinonen-Kilpeläinen-Martio [13] (they call it cap $_{p}$ ).

Theorem 2.2. (Theorem 6.3 in A. Björn [2]) Let $E \subset \Omega$ be a relatively closed set with $C_{p}(E)=0$. Assume that $u$ is bounded from below and $Q$-quasisuperharmonic in $\Omega \backslash E$. Then $u$ has a $Q$-quasisuperharmonic extension $U$ to $\Omega$ given by $U(x)=$ $\operatorname{ess~liminf}_{\Omega \backslash E \ni y \rightarrow x} u(y)$.

For $Q=1$ and weighted $\mathbf{R}^{n}$ this is Theorem 7.35 in Heinonen-KilpeläinenMartio [13].

We want to study radially symmetric functions, primarily powers, and determine when they are quasiminimizers. The following result is important to clarify which conditions we should discuss.

Proposition 2.3. Let $u(x)=|x|^{\alpha}$. Then the following implications and equivalences hold for $u$ :

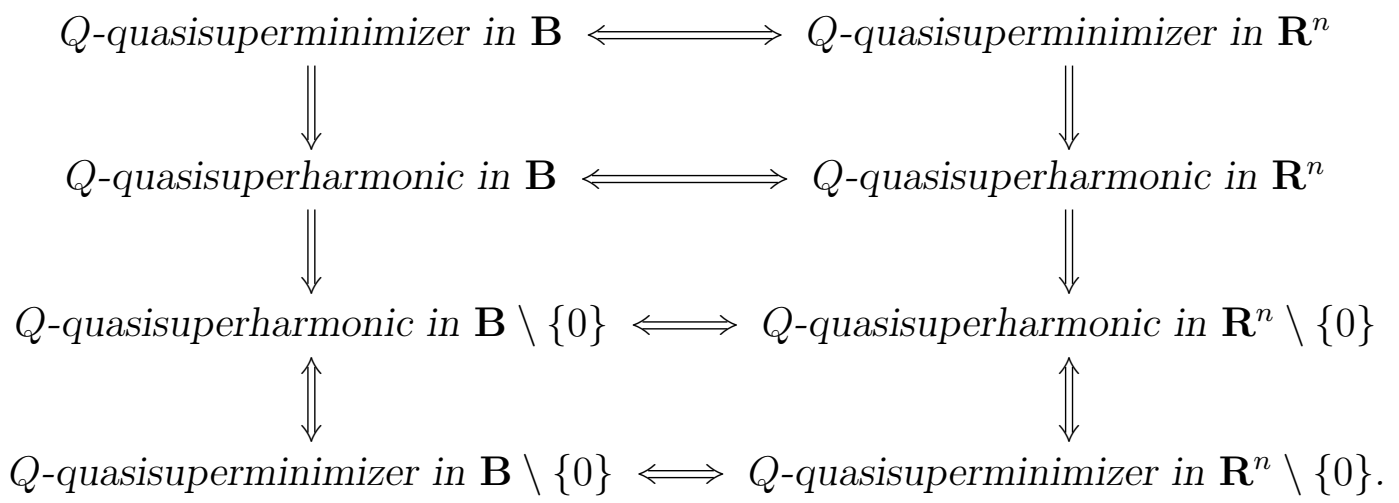

The above implications remain true if "super" is replaced by "sub". Moreover, $u$ is a $Q$-quasisubminimizer in B if and only if it is $Q$-quasisubharmonic there, i.e. the uppermost downward directed implication is an equivalence in this case.

In view of this we will concentrate on discussing the first, second and fourth condition in the left column for quasisuperminimizers and the conditions corresponding to the first and fourth condition in the left column for quasisubminimizers.

Remark 2.4. (i) For $1<p \leq n$, the middle downwards implications for the super case are equivalences, by Theorem 2.2 , as $C_{p}(\{0\})=0$.

(ii) For $p=n$ we will also consider powers of $\log$, i.e. $u(x)=(-\log |x|)^{\alpha}$, in which case the left column holds (with the same proof, and including the equivalence in the middle as in (i)), while $u$, for most $\alpha$, is not even defined for $|x|>1$. Similarly when "super" is replaced by "sub", we have the same implications in the left column as for powers.

(iii) Note also that the proof shows that the left (resp. right) column in Proposition 2.3 holds for every extended real-valued function which is continuous in $\mathbf{B}$ (resp. $\mathbf{R}^{n}$ ) and locally bounded in $\mathbf{B} \backslash\{0\}$ (resp. $\left.\mathbf{R}^{n} \backslash\{0\}\right)$. 
Proof. The lowest downwards-directed implications follow directly from the definition and the fact that $u$ is locally bounded in $\mathbf{R}^{n} \backslash\{0\}$. The other vertical implications are trivially true for arbitrary extended real-valued continuous functions.

The left-directed horizontal implications are trivial. Let us prove the top rightdirected horizontal implication. Assume that $u$ is a $Q$-quasisuperminimizer in B. Let $\widetilde{\varphi} \in \operatorname{Lip}_{c}\left(\mathbf{R}^{n}\right)$ be nonnegative. Then there is $R>0$ such that $\widetilde{\varphi} \in \operatorname{Lip}_{c}(B(0, R))$. Let $v(x)=u(R x)$ and $\varphi(x)=\widetilde{\varphi}(R x)$. Then $v=R^{\alpha} u$ and thus $v$ is a $Q$-quasisuperminimizer in B. As $\varphi \in \operatorname{Lip}_{c}(\mathbf{B})$, we have

$$
\begin{aligned}
\int_{\widetilde{\varphi} \neq 0}|\nabla u|^{p} d x & =R^{n-p} \int_{\varphi \neq 0}|\nabla v|^{p} d x \\
& \leq Q R^{n-p} \int_{\varphi \neq 0}|\nabla(v+\varphi)|^{p} d x=Q \int_{\widetilde{\varphi} \neq 0}|\nabla(u+\widetilde{\varphi})|^{p} d x .
\end{aligned}
$$

Hence $u$ is a $Q$-quasisuperminimizer in $\mathbf{R}^{n}$.

The other right-directed horizontal implications are proved similarly, using in addition Definition 2.1 for those implications concerning quasisuperharmonicity.

The proofs in the "sub" case are similar. Moreover, if $u$ is $Q$-quasisubharmonic in $\mathbf{B}$, then $u$ is also a $Q$-quasisubminimizer in $\mathbf{B}$, as $u>0$ is bounded from below.

\section{Sub- and superminimizers}

The results in this section are straightforward and well known to experts but may not all have been recorded explicitly in the literature. We will need them for the later parts of the paper. Note that the statements in Theorems 3.1 and 3.2 can be read off from Tables 1-3, and the results in Theorems 3.3 and 3.4 can be read off from Table 4.

\subsection{Powers.}

Theorem 3.1. Let $u(x)=|x|^{\alpha}$. Then $u$ is a superminimizer in $\mathbf{B} \backslash\{0\}$ if and only if

$$
\begin{cases}\frac{p-n}{p-1} \leq \alpha \leq 0, & \text { if } 1<p<n \\ \alpha=0, & \text { if } p=n \\ 0 \leq \alpha \leq \frac{p-n}{p-1}, & \text { if } p>n\end{cases}
$$

Similarly, $u$ is a subminimizer in $\mathbf{B} \backslash\{0\}$ if and only if

$$
\begin{cases}\alpha \leq \frac{p-n}{p-1} \text { or } \alpha \geq 0, & \text { if } 1<p<n, \\ \alpha \text { is arbitrary, } & \text { if } p=n, \\ \alpha \leq 0 \text { or } \alpha \geq \frac{p-n}{p-1}, & \text { if } p>n .\end{cases}
$$

Proof. A straightforward calculation shows that

$$
\operatorname{div}\left(|\nabla u(x)|^{p-2} \nabla u(x)\right)=\alpha\left(\alpha-\frac{p-n}{p-1}\right)(p-1)|\alpha|^{p-2}|x|^{\alpha(p-1)-p} \quad \text { for } x \in \mathbf{B} \backslash\{0\} .
$$

The function $u$ is, by definition, a subminimizer if this expression is nonnegative, and a superminimizer if it is nonpositive throughout $\mathbf{B} \backslash\{0\}$, and it is easy to check that this happens exactly as stated. 
Theorem 3.2. Let $u(x)=|x|^{\alpha}$. Then $u$ is superharmonic in $\mathbf{B}$ if and only if

$$
\begin{cases}\frac{p-n}{p-1} \leq \alpha \leq 0, & \text { if } 1<p<n, \\ \alpha=0, & \text { if } p \geq n .\end{cases}
$$

Moreover, $u$ is a superminimizer in $\mathbf{B}$ if and only if

$$
\begin{cases}1-\frac{n}{p}<\alpha \leq 0, & \text { if } 1<p<n, \\ \alpha=0, & \text { if } p \geq n .\end{cases}
$$

Similarly, $u$ is subharmonic (or equivalently a subminimizer) in B if and only if

$$
\begin{cases}\alpha \geq 0, & \text { if } 1<p \leq n, \\ \alpha=0 \text { or } \alpha \geq \frac{p-n}{p-1}, & \text { if } p>n .\end{cases}
$$

Proof. The case $\alpha=0$ is clear as $u$ is constant in this case. If $\alpha>0$, then $u$ is not superharmonic in $\mathbf{B}$ as it would violate the minimum principle.

If $1<p<n$ and $(p-n) /(p-1) \leq \alpha \leq 0$, then $u$ is superharmonic in $\mathbf{B} \backslash\{0\}$, by Theorem 3.1, and thus in $\mathbf{B}$, by Remark 2.4(i) (or Theorem 2.2). In this case, $u$ is a superminimizer in $\mathbf{B}$ if and only if $u \in W_{\text {loc }}^{1, p}(\mathbf{B})$, i.e. if and only if $\alpha>1-n / p$. That $u$ is neither a superminimizer nor superharmonic in the remaining cases follows directly from Theorem 3.1.

For subharmonicity, note first that if $\alpha<0$, then $u(0)=\infty$ and thus $u$ is not subharmonic in B. For $1<p \leq n$ and $\alpha \geq 0$, it follows from Theorem 3.1 that $u$ is subharmonic in $\mathbf{B} \backslash\{0\}$ and thus in $\mathbf{B}$, by Theorem 2.2, and a subminimizer in $\mathbf{B}$, by Proposition 2.3 .

For $p>n$, the case $0<\alpha<(p-n) /(p-1)$ follows from Theorem 3.1. So assume that $p>n$ and $\alpha \geq(p-n) /(p-1)$. In this case $u$ is a subminimizer in $\mathbf{B} \backslash\{0\}$ by Theorem 3.1. Moreover $u \in W_{\text {loc }}^{1, p}(\mathbf{B})$. Let $\varphi \in \operatorname{Lip}_{c}(\mathbf{B})$ be nonpositive and $\widetilde{\varphi}=\max \{\varphi,-u\}$ so that $u+\widetilde{\varphi}=(u+\varphi)_{+}$and $|\nabla(u+\widetilde{\varphi})| \leq|\nabla(u+\varphi)|$. Then $\widetilde{\varphi} \in W_{0}^{1, p}(\mathbf{B} \backslash\{0\})$ and hence

$$
\int_{\varphi \neq 0}|\nabla u|^{p} d x=\int_{\widetilde{\varphi} \neq 0}|\nabla u|^{p} d x \leq \int_{\widetilde{\varphi} \neq 0}|\nabla(u+\widetilde{\varphi})|^{p} d x \leq \int_{\varphi \neq 0}|\nabla(u+\varphi)|^{p} d x .
$$

Thus $u$ is a subminimizer, and hence subharmonic, in $\mathbf{B}$.

\section{2. log-powers for $p=n$.}

Theorem 3.3. Let $p=n$ and $u(x)=(-\log |x|)^{\alpha}$. Then $u$ is a superminimizer in $\mathbf{B} \backslash\{0\}$ if and only if $0 \leq \alpha \leq 1$. Moreover, $u$ is a subminimizer in $\mathbf{B} \backslash\{0\}$ if and only if $\alpha \leq 0$ or $\alpha \geq 1$.

Proof. A straightforward calculation shows that

$$
\operatorname{div}\left(|\nabla u(x)|^{n-2} \nabla u(x)\right)=\alpha(\alpha-1)|\alpha|^{n-2}(n-1)(-\log |x|)^{(n-1) \alpha-n}|x|^{-n}
$$

for $x \in \mathbf{B} \backslash\{0\}$. The sign of this expression is the same as of $\alpha(\alpha-1)$, which is nonpositive if and only if $0 \leq \alpha \leq 1$, i.e. $u$ is a superminimizer if and only if $0 \leq \alpha \leq 1$. Similarly, $u$ is a subminimizer if and only if $\alpha(\alpha-1) \geq 0$, i.e. if and only if $\alpha \leq 0$ or $\alpha \geq 1$. 
Theorem 3.4. Let $p=n$ and $u(x)=(-\log |x|)^{\alpha}$. Then $u$ is subharmonic (or equivalently a subminimizer) in $\mathbf{B}$ if and only if $\alpha \leq 0$.

Similarly, $u$ is superharmonic in $\mathbf{B}$ if and only if $0 \leq \alpha \leq 1$, and it is a superminimizer in $\mathbf{B}$ if and only if $0 \leq \alpha<1-1 / n$.

Proof. The first part follows from Theorems 2.2 and 3.3, together with the fact that $u(0)=\infty$ if $\alpha>0$, and thus $u$ is not subharmonic (nor a subminimizer) in $\mathbf{B}$ for $\alpha>0$.

The second part follows from Theorems 2.2 and 3.3, together with the fact that a superharmonic function in $\mathbf{B}$ is a superminimizer in $\mathbf{B}$ if and only if it belongs to $W_{\text {loc }}^{1, p}(\mathbf{B})$, which for our $u$ holds exactly if $\alpha<1-1 / n$.

\section{Power-type quasiminimizers}

We want to study radially symmetric functions, primarily powers, and determine when they are quasiminimizers. Let us introduce some notation. Let $\varphi:(0, \infty) \rightarrow \mathbf{R}$ be given and let $u(x)=\varphi(|x|)$ be a radially symmetric function. Then $|\nabla u(x)|=$ $\left|\varphi^{\prime}(|x|)\right|$. Sometimes we consider $u$ defined also at 0 , in which case we define $u(0)=$ $\lim _{x \rightarrow 0} u(x)$.

Let for the moment $\Omega=\left\{x: r_{1}<|x|<r_{2}\right\}$ and $G=\left\{r: r_{1}<r<r_{2}\right\}$, $0<r_{1}<r_{2}$. We want to calculate the $p$-energy of $u$, viz.

$$
I_{u}(\Omega):=\int_{\Omega}|\nabla u|^{p} d x=c_{n-1} \int_{r_{1}}^{r_{2}}\left|\varphi^{\prime}\right|^{p} r^{n-1} d r=: c_{n-1} \hat{I}_{\varphi}(G),
$$

where $c_{n-1}$ is the surface area of the sphere $\mathbf{S}^{n-1}$ (if $n=1$ we have $c_{0}=2$ ).

We want to compare the energy $I_{u}$ with the energy of the minimizer, the $p$ harmonic function, $v$ having the same boundary values on $\partial \Omega$. It is well known that the function $w$ given by

$$
w(x)=\psi(|x|), \quad \psi(r)= \begin{cases}r^{(p-n) /(p-1)}, & \text { if } p \neq n, \\ \log r, & \text { if } p=n,\end{cases}
$$

is $p$-harmonic in $\mathbf{R}^{n} \backslash\{0\}$. It follows that $v=a w+b$ for some appropriately chosen $a, b \in \mathbf{R}$.

Since we know that $w$ minimizes the energy $I$ given its boundary values on $\partial \Omega$, it follows that $\psi$ minimizes the energy $\hat{I}$ given its boundary values $\psi\left(r_{1}\right)$ and $\psi\left(r_{2}\right)$ on $\partial G$. We will use this fact.

Theorem 4.1. Let $1<p \neq n, \alpha \neq 0, \alpha \neq 1-n / p$ and $u(x)=|x|^{\alpha}$. Then $u$ is a quasiminimizer in $\mathbf{B} \backslash\{0\}$ if and only if

$$
M:=\limsup _{R \rightarrow \infty}\left|\frac{1-R^{\beta}}{1-R^{\alpha}}\right|^{p}\left|\frac{R^{p \alpha-p+n}-1}{R^{p \beta-p+n}-1}\right|<\infty,
$$

where $\beta=(p-n) /(p-1)$.

Moreover, if (4.1) holds, then

$$
Q=\left|\frac{\alpha}{\beta}\right|^{p}\left|\frac{p \beta-p+n}{p \alpha-p+n}\right| M
$$

is the best quasiminimizer constant for $u$. 
Proof. Let $\varphi(r)=r^{\alpha}$, and let us calculate $\hat{I}_{\varphi}(G)$, where $G=\left\{r: r_{1}<r<r_{2}\right\}$, $0<r_{1}<r_{2}<1$. Note that $p \alpha-p+n \neq 0$, since $\alpha \neq 1-n / p$.

We get, with $R=r_{2} / r_{1}>1$,

$$
\begin{aligned}
\hat{I}_{\varphi}(G) & =\int_{r_{1}}^{r_{2}}\left|\alpha r^{\alpha-1}\right|{ }^{p} r^{n-1} d r=\frac{|\alpha|^{p}}{p \alpha-p+n}\left(r_{2}^{p \alpha-p+n}-r_{1}^{p \alpha-p+n}\right) \\
& =\frac{|\alpha|^{p}}{p \alpha-p+n} r_{1}^{p \alpha-p+n}\left(R^{p \alpha-p+n}-1\right) .
\end{aligned}
$$

For the minimizer of the $\hat{I}$-energy we let $\psi(r)=r^{\beta}$. Putting $\alpha=\beta$ in the calculation above we see that

$$
\hat{I}_{\psi}(G)=\frac{|\beta|^{p}}{p \beta-p+n} r_{1}^{p \beta-p+n}\left(R^{p \beta-p+n}-1\right) .
$$

Actually, $p \beta-p+n=\beta$, but let us keep the expression as it is.

Let now $\eta=a \psi+b$, where we choose $a$ and $b$ so that $\eta=\varphi$ on $\partial G$, i.e. so that

$$
r_{1}^{\alpha}=a r_{1}^{\beta}+b \quad \text { and } \quad r_{2}^{\alpha}=a r_{2}^{\beta}+b
$$

In fact we only need to determine

$$
a=\frac{r_{1}^{\alpha}-r_{2}^{\alpha}}{r_{1}^{\beta}-r_{2}^{\beta}} .
$$

Thus

$$
\hat{I}_{\eta}(G)=|a|^{p} \hat{I}_{\psi}(G)
$$

and we know that $\eta$ minimizes the $\hat{I}$-energy given the boundary values of $\varphi$ on $\partial G$.

Next we want to calculate

$$
Q:=\sup _{0<r_{1}<r_{2}<1} \frac{\hat{I}_{\varphi}(G)}{\hat{I}_{\eta}(G)} .
$$

We will show that this $Q$, if finite, is the best quasiminimizer constant for $u$ on $\mathbf{B} \backslash\{0\}$. Comparing $u$ with $x \mapsto \eta(|x|)$ shows directly that we cannot have a quasiminimizer constant for $u$ less than $Q$ above. In particular if $Q=\infty$, then it follows directly that $u$ is not a quasiminimizer.

We have, still letting $R=r_{2} / r_{1}$,

$$
\begin{aligned}
\frac{\hat{I}_{\varphi}(G)}{\hat{I}_{\eta}(G)} & =\left|\frac{\alpha}{\beta}\right|^{p} \frac{p \beta-p+n}{p \alpha-p+n}\left|\frac{r_{1}^{\beta}-r_{2}^{\beta}}{r_{1}^{\alpha}-r_{2}^{\alpha}}\right|^{p} r_{1}^{p(\alpha-\beta)} \frac{R^{p \alpha-p+n}-1}{R^{p \beta-p+n}-1} \\
& =\left|\frac{\alpha}{\beta}\right|^{p} \frac{p \beta-p+n}{p \alpha-p+n}\left|\frac{1-R^{\beta}}{1-R^{\alpha}}\right|^{p} \frac{R^{p \alpha-p+n}-1}{R^{p \beta-p+n}-1}=: k(R) .
\end{aligned}
$$

In particular we see that $\hat{I}_{\varphi}(G) / \hat{I}_{\eta}(G)$ only depends on $R$. Let $r=\sqrt{r_{1} r_{2}}$ and let $\eta_{1}$ and $\eta_{2}$ be the minimizers of the $\hat{I}$-energy on $G_{1}=\left(r_{1}, r\right)$ and $G_{2}=\left(r, r_{2}\right)$, respectively, i.e. $\eta_{i}=a_{i} \psi+b_{i}$ such that $r_{i}^{\alpha}=a_{i} r_{i}^{\beta}+b_{i}$ and $r^{\alpha}=a_{i} r^{\beta}+b_{i}, i=1,2$. Let further $\tilde{\eta}=\eta_{1} \chi_{G_{1}}+\eta_{2} \chi_{(0,1) \backslash G_{1}}$. Then, as $r / r_{1}=r_{2} / r=\sqrt{R}$, we have

$$
\begin{aligned}
\hat{I}_{\varphi}(G) & =k(R) \hat{I}_{\eta}(G) \leq k(R) \hat{I}_{\tilde{\eta}}(G)=k(R)\left(\hat{I}_{\eta_{1}}\left(G_{1}\right)+\hat{I}_{\eta_{2}}\left(G_{2}\right)\right) \\
& =k(R)\left(\frac{\hat{I}_{\varphi}\left(G_{1}\right)}{k(\sqrt{R})}+\frac{\hat{I}_{\varphi}\left(G_{2}\right)}{k(\sqrt{R})}\right)=\frac{k(R)}{k(\sqrt{R})} \hat{I}_{\varphi}(G) .
\end{aligned}
$$


As $\hat{I}_{\varphi}(G) \neq 0$, we find that $k(R) \geq k(\sqrt{R})$, from which it follows that $Q=$ $\sup _{R>1} k(R)=\limsup _{R \rightarrow \infty} k(R)$, which is clearly finite if and only if (4.1) holds.

Finally, we show that if $Q<\infty$ then $u$ is indeed a $Q$-quasiminimizer. Let $\omega$ be such that $\omega-\varphi \in \operatorname{Lip}_{c}((0,1))$. The open set $V=\{x \in(0,1): \omega(x) \neq \varphi(x)\}$ can be written as a countable (or finite) union of intervals $\left\{I_{j}\right\}_{j}$. We find that

$$
\hat{I}_{\varphi}(V)=\sum_{j} \hat{I}_{\varphi}\left(I_{j}\right) \leq \sum_{j} Q \hat{I}_{\omega}\left(I_{j}\right)=Q \hat{I}_{\omega}(V)
$$

Hence $\varphi$ is indeed a $Q$-quasiminimizer for the energy $\hat{I}$ on $(0,1)$.

Let us finally turn to $u$. Let $v$ be such that $v-u \in \operatorname{Lip}_{c}(\mathbf{B} \backslash\{0\})$. Let further $\Omega=\{x \in \mathbf{B} \backslash\{0\}: v(x) \neq u(x)\}$. Using polar coordinates $x=(r, \theta)$, where $0<r<1$ and $\theta \in \mathbf{S}^{n-1}$, let $V_{\theta}=\{r:(r, \theta) \in \Omega\}$ and $v_{\theta}(r)=v(r, \theta)$. We then find, applying (4.3) to $G=V_{\theta}$, that

$$
\begin{aligned}
I_{u}(\Omega) & =\int_{\mathbf{S}^{n-1}} \hat{I}_{\varphi}\left(V_{\theta}\right) d \theta \leq \int_{\mathbf{S}^{n-1}} Q \hat{I}_{v_{\theta}}\left(V_{\theta}\right) d \theta \\
& =Q \int_{\Omega}\left|\frac{\partial v}{\partial r}\right|^{p} d x \leq Q \int_{\Omega}|\nabla v|^{p} d x=Q I_{v}(\Omega) .
\end{aligned}
$$

Hence $u$ is a $Q$-quasiminimizer in $\mathbf{B} \backslash\{0\}$.

Next, we take care of the case $\alpha=1-n / p$, which was omitted in Theorem 4.1.

Proposition 4.2. Let $1<p \neq n, \alpha=1-n / p$ and $u(x)=|x|^{\alpha}$. Then $u$ is not a quasiminimizer in $\mathbf{B} \backslash\{0\}$.

Proof. As in the proof of Theorem 4.1, we find that

$$
\hat{I}_{\varphi}(G)=|\alpha|^{p}\left(\log r_{2}-\log r_{1}\right)=|\alpha|^{p} \log R,
$$

where $R=r_{2} / r_{1}$. With $\beta=(p-n) /(p-1)$ and $\eta$ as in the proof of Theorem 4.1 we get, using that $p \beta-p+n=\beta=p(\beta-\alpha)$,

$$
\begin{aligned}
\frac{\hat{I}_{\varphi}(G)}{\hat{I}_{\eta}(G)} & =\left|\frac{\alpha}{\beta}\right|^{p}(p \beta-p+n)\left|\frac{r_{1}^{\beta}-r_{2}^{\beta}}{r_{1}^{\alpha}-r_{2}^{\alpha}}\right|^{p} r_{1}^{-(p \beta-p+n)} \frac{\log R}{R^{p \beta-p+n}-1} \\
& =\left|\frac{\alpha}{\beta}\right|^{p} \beta\left|\frac{1-R^{\beta}}{1-R^{\alpha}}\right|^{p} \frac{\log R}{R^{\beta}-1}=: k(R) .
\end{aligned}
$$

Depending on whether $p<n$ or $p>n$, we see that $\alpha$ and $\beta$ are either both negative or both positive and hence $k(R)$ grows as $\log R$, as $R \rightarrow \infty$, showing that $u$ is not a quasiminimizer.

\section{The case $1<p<n$}

Theorem 5.1. Let $1<p<n$ and $u(x)=|x|^{\alpha}$. Then $u$ is a quasiminimizer in $\mathbf{B} \backslash\{0\}$ if and only if $\alpha<1-n / p$ or $\alpha=0$.

Moreover, if $\alpha<1-n / p$, then

$$
Q_{\alpha, p, n}=\left(\frac{\alpha}{\beta}\right)^{p} \frac{p \beta-p+n}{p \alpha-p+n}
$$

is the best quasiminimizer constant, where $\beta=(p-n) /(p-1)$.

In fact, $p \beta-p+n=\beta$ so that $Q=|\alpha|^{p} /|\beta|^{p-1}|p \alpha-p+n|$. 
Remark 5.2. It is sometimes interesting to determine $\alpha$ in terms of $Q$. In general this seems impossible, but for $p=2<n$ it is easy to see that

$$
\alpha=(n-2)\left(-Q \pm \sqrt{Q^{2}-Q}\right) .
$$

However, noting that $p \alpha<p \alpha-p+n<\alpha$ for $\alpha<\beta$, we easily obtain the following estimate for $Q>1$ and $\alpha<\beta$,

$$
(p Q)^{1 /(p-1)} \beta<\alpha<Q^{1 /(p-1)} \beta .
$$

Proof of Theorem 5.1. The case $\alpha=0$ is clear as $u$ is constant in this case. The case $\alpha=1-n / p$ follows from Proposition 4.2. Next, we shall use Theorem 4.1. Note that $\beta=p \beta-p+n<0$ here.

Case 1. $\alpha<1-n / p$. Then $p \alpha-p+n<0$ and

$$
\lim _{R \rightarrow \infty}\left|\frac{1-R^{\beta}}{1-R^{\alpha}}\right|^{p}\left|\frac{R^{p \alpha-p+n}-1}{R^{p \beta-p+n}-1}\right|=1 .
$$

Thus (4.1) holds and $Q_{\alpha, p, n}$ is the best quasiminimizer constant, by Theorem 4.1.

Case 2. $1-n / p<\alpha<0$. Then $p \alpha-p+n>0$, and the expression in (4.1) grows as $R^{p \alpha-p+n}$, as $R \rightarrow \infty$. Hence $u$ is not a quasiminimizer.

Case 3. $\alpha>0$. Then $p \alpha-p+n>0$ and the expression in (4.1) grows as $R^{n-p}$, as $R \rightarrow \infty$. Hence $u$ is not a quasiminimizer.

Theorem 5.3. Let $1<p<n, u(x)=|x|^{\alpha}$ and let $Q_{\alpha, p, n}$ be as in (5.1). Then $u$ is a $Q$-quasi(sub/super)minimizer and $Q$-quasi(sub/super)harmonic in $\mathbf{B} \backslash\{0\}$ and $\mathbf{B}$ as given in Table 1. Moreover, $Q$ in Table 1 is the best quasi(sub/super)minimizer constant.

\begin{tabular}{|c||c|c|c|c|c|}
\hline $1<p<n$ & $\begin{array}{c}\text { quasi- } \\
\text { sub- } \\
\text { mini- } \\
\text { mizer } \\
\text { in } \mathbf{B} \backslash\{0\}\end{array}$ & $\begin{array}{c}\text { quasi- } \\
\text { sub- } \\
\text { mini- } \\
\text { mizer } \\
\text { in B }\end{array}$ & $\begin{array}{c}\text { quasi- } \\
\text { super- } \\
\text { mini- } \\
\text { mizer } \\
\text { in B }\end{array}$ & $\begin{array}{c}\text { quasi- } \\
\text { super- } \\
\text { mini- } \\
\text { mizer } \\
\text { in } \mathbf{B} \backslash\{0\}\end{array}$ & $\begin{array}{c}\text { quasi- } \\
\text { super- } \\
\text { harmo- } \\
\text { nic } \\
\text { in B }\end{array}$ \\
\hline \hline$\alpha<\frac{p-n}{p-1}$ & $Q=1$ & Fails & Fails & $Q=Q_{\alpha, p, n}$ & $Q=Q_{\alpha, p, n}$ \\
\hline$\alpha=\frac{p-n}{p-1}$ & $Q=1$ & Fails & Fails & $Q=1$ & $Q=1$ \\
\hline$\frac{p-n}{p-1}<\alpha<1-\frac{n}{p}$ & $Q=Q_{\alpha, p, n}$ & Fails & Fails & $Q=1$ & $Q=1$ \\
\hline$\alpha=1-\frac{n}{p}$ & Fails & Fails & Fails & $Q=1$ & $Q=1$ \\
\hline$\frac{n}{p}<\alpha<0$ & Fails & Fails & $Q=1$ & $Q=1$ & $Q=1$ \\
\hline$\alpha=0$ & $Q=1$ & $Q=1$ & $Q=1$ & $Q=1$ & $Q=1$ \\
\hline$\alpha>0$ & $Q=1$ & $Q=1$ & Fails & Fails & Fails \\
\hline
\end{tabular}

Table 1. 
Remark 5.4. Recall that, by Proposition 2.3, $|x|^{\alpha}$ is a $Q$-quasisubminimizer in $\mathbf{B} \backslash\{0\}$ or $\mathbf{B}$ if and only if it is $Q$-quasisubharmonic there, and $u$ is a $Q$ quasisuperminimizer in $\mathbf{B} \backslash\{0\}$ if and only if it is $Q$-quasisuperharmonic there.

Recall also that Remark 2.4(i) shows that the last two columns are the same in this case.

Proof. Note first that the case $\alpha=0$ is clear as $u$ is constant in this case. Also, if $\alpha=(p-n) /(p-1)$ then $Q_{\alpha, p, n}=1$. Moreover, the last two columns are the same by Remark $2.4(\mathrm{i})$.

Case 1. $\alpha \leq(p-n) /(p-1)$. By Theorem 5.1, $u$ is a quasiminimizer in $\mathbf{B} \backslash\{0\}$ with $Q_{\alpha, p, n}$ being the best quasiminimizer constant. As $u$ is a subminimizer in $\mathbf{B} \backslash\{0\}$, by Theorem 3.1, $Q_{\alpha, p, n}$ must be the best quasisuperminimizer constant in $\mathbf{B} \backslash\{0\}$. As $u \notin W_{\text {loc }}^{1, p}(\mathbf{B})$ it is neither a quasisubminimizer nor a quasisuperminimizer in $\mathbf{B}$.

Case 2. $(p-n) /(p-1)<\alpha<0$. In this case $u$ is superharmonic in $\mathbf{B}$, by Theorem 3.2. As $u \in W_{\mathrm{loc}}^{1, p}(\mathbf{B})$ if and only if $\alpha>1-n / p, u$ is a quasisuperminimizer in $\mathbf{B}$ only for such $\alpha$. By Theorem 5.1, $u$ is a quasiminimizer in $\mathbf{B} \backslash\{0\}$ if and only if $\alpha<1-n / p$, with $Q_{\alpha, p, n}$ being the best quasiminimizer constant. As $u$ is a superminimizer in $\mathbf{B} \backslash\{0\}$, it follows that $Q_{\alpha, p, n}$ is the best quasisubminimizer constant for $\alpha<1-n / p$, and that $u$ fails to be a quasisubminimizer in $\mathbf{B} \backslash\{0\}$ for $1-n / p \leq \alpha<0$. As $u(0)=\infty, u$ cannot be quasisubharmonic (and thus not a quasisubminimizer) in $\mathbf{B}$.

Case 3. $\alpha>0$. By Theorem 3.2, $u$ is a subminimizer in $\mathbf{B}$ and thus in $\mathbf{B} \backslash\{0\}$. On the other hand, $u$ is not a quasiminimizer in $\mathbf{B} \backslash\{0\}$, by Theorem 5.1. Thus $u$ cannot be a quasisuperminimizer in $\mathbf{B} \backslash\{0\}$ and thus not in $\mathbf{B}$ either.

\section{The case $p>n$}

Note that in this case $n=1$ is a possibility.

Theorem 6.1. Let $p>n$ and $u(x)=|x|^{\alpha}$. Then $u$ is a quasiminimizer in $\mathbf{B} \backslash\{0\}$ if and only if $\alpha>1-n / p$ or $\alpha=0$.

Moreover, if $\alpha>1-n / p$, then $Q_{\alpha, p, n}$ given by (5.1) is the best quasiminimizer constant.

For $n=1$ and $p=2$ this result was obtained by Judin [14], Example 4.0.26 and Remark 4.0.28, and Martio [18], Section 5. The formula given in Remark 5.2 for $\alpha$ in terms of $Q$ when $p=2$ is valid also in this case, i.e. when $n=1$ and $p=2$.

Proof. The case $\alpha=0$ is clear as $u$ is constant in this case. The case $\alpha=$ $1-n / p$ follows from Proposition 4.2. Next, we shall use Theorem 4.1. Note that $\beta=p \beta-p+n>0$ here.

Case 1. $\alpha>1-n / p>0$. In this case $p \alpha-p+n>0$ and hence

$$
\lim _{R \rightarrow \infty}\left|\frac{1-R^{\beta}}{1-R^{\alpha}}\right|^{p}\left|\frac{R^{p \alpha-p+n}-1}{R^{p \beta-p+n}-1}\right|=1 .
$$

Thus (4.1) holds, $u$ is a quasiminimizer in $\mathbf{B} \backslash\{0\}$ and $Q_{\alpha, p, n}$ is the best quasiminimizer constant.

Case 2. $0<\alpha<1-n / p$. In this case $p \alpha-p+n<0$ and the expression in (4.1) grows as $R^{p-n-p \alpha}$, as $R \rightarrow \infty$. It follows that $u$ is not a quasiminimizer in $\mathbf{B} \backslash\{0\}$. 
Case 3. $\alpha<0$. In this case $p \alpha-p+n<0$ and the expression in (4.1) grows as $R^{p-n}$, as $R \rightarrow \infty$. Thus $u$ is not a quasiminimizer in $\mathbf{B} \backslash\{0\}$.

Theorem 6.2. Let $p>n, u(x)=|x|^{\alpha}$ and let $Q_{\alpha, p, n}$ be as in (5.1). Then $u$ is a $Q$-quasi(sub/super)minimizer and $Q$-quasi(sub/super)harmonic in $\mathbf{B} \backslash\{0\}$ and $\mathbf{B}$ as given in Table 2. Moreover, $Q$ in Table 2 is the best quasi(sub/super)minimizer constant.

\begin{tabular}{|c||c|c|c|c|c|}
\hline \multicolumn{1}{|c||}{$p>n$} & $\begin{array}{c}\text { quasi- } \\
\text { sub- } \\
\text { mini- } \\
\text { mizer } \\
\text { in } \mathbf{B} \backslash\{0\}\end{array}$ & $\begin{array}{c}\text { quasi- } \\
\text { sub- } \\
\text { mini- } \\
\text { mizer } \\
\text { in B }\end{array}$ & $\begin{array}{c}\text { quasi- } \\
\text { super- } \\
\text { mini- } \\
\text { mizer } \\
\text { in B }\end{array}$ & $\begin{array}{c}\text { quasi- } \\
\text { super- } \\
\text { mini- } \\
\text { mizer } \\
\text { in } \mathbf{B} \backslash\{0\}\end{array}$ & $\begin{array}{c}\text { quasi- } \\
\text { super- } \\
\text { harmo- } \\
\text { nic } \\
\text { in B }\end{array}$ \\
\hline \hline$\alpha<0$ & $Q=1$ & Fails & Fails & Fails & Fails \\
\hline$\alpha=0$ & $Q=1$ & $Q=1$ & $Q=1$ & $Q=1$ & $Q=1$ \\
\hline $0<\alpha \leq 1-\frac{n}{p}$ & Fails & Fails & Fails & $Q=1$ & Fails \\
\hline $1-\frac{n}{p}<\alpha<\frac{p-n}{p-1}$ & $Q=Q_{\alpha, p, n}$ & $Q=Q_{\alpha, p, n}$ & Fails & $Q=1$ & Fails \\
\hline$\alpha=\frac{p-n}{p-1}$ & $Q=1$ & $Q=1$ & Fails & $Q=1$ & Fails \\
\hline$\alpha>\frac{p-n}{p-1}$ & $Q=1$ & $Q=1$ & Fails & $Q=Q_{\alpha, p, n}$ & Fails \\
\hline
\end{tabular}

Table 2 .

Remark 6.3. Recall that, by Proposition 2.3, $|x|^{\alpha}$ is a $Q$-quasisubminimizer in $\mathbf{B} \backslash\{0\}$ or $\mathbf{B}$ if and only if it is Q-quasisubharmonic there, and $u$ is a $Q$ quasisuperminimizer in $\mathbf{B} \backslash\{0\}$ if and only if it is $Q$-quasisuperharmonic there.

Moreover, Theorem 6.2 now shows that for $p>n,|x|^{\alpha}$ is $Q$-quasisuperharmonic in $\mathbf{B}$ if and only if it is a $Q$-quasisuperminimizer in $\mathbf{B}$. This is not very surprising, in fact for $\alpha \geq 0, u$ is quasisuperharmonic in $\mathbf{B}$ if and only if $u$ is a quasisuperminimizer in $\mathbf{B}$, as $u$ is bounded in this case. On the other hand, if $\alpha<0$, then $u$ is not quasisuperharmonic in $\mathbf{B}$ (and hence cannot be a quasisuperminimizer in $\mathbf{B}$ ) as $u(0)=\infty$ and $C_{p}(\{0\})>0$, and a quasisuperharmonic function is infinite only in a set with zero capacity, by Kinnunen-Martio [15], Theorem 10.6.

Proof. The case $\alpha=0$ is clear as $u$ is constant in this case.

Case 1. $\alpha<0$. By Theorem 3.1, $u$ is a subminimizer in $\mathbf{B} \backslash\{0\}$. As $u(0)=$ $\infty, u$ cannot be quasisubharmonic (and thus not a quasisubminimizer) in $\mathbf{B}$. By Theorem 6.1, $u$ is not a quasiminimizer in $\mathbf{B} \backslash\{0\}$. As it is a subminimizer in $\mathbf{B} \backslash\{0\}$, it cannot be a quasisuperminimizer (and thus not quasisuperharmonic) there (and not in $\mathbf{B}$ either).

Case 2. $\alpha>0$. In this case $u$ is not quasisuperharmonic (and thus not a quasisuperminimizer) in $\mathbf{B}$ as it would violate the strong minimum principle.

For $0<\alpha \leq(p-n) /(p-1), u$ is a superminimizer in $\mathbf{B} \backslash\{0\}$, by Theorem 3.1. For $\alpha \geq(p-n) /(p-1), u$ is a subminimizer in $\mathbf{B}$ (and thus also in $\mathbf{B} \backslash\{0\}$ ) by Theorem 3.2. 
Theorem 6.1 shows that $u$ is a quasiminimizer in $\mathbf{B} \backslash\{0\}$ if and only if $\alpha>$ $1-n / p$, in which case $Q_{\alpha, p, n}$ is the best quasiminimizer constant. For $1-n / p<$ $\alpha<(p-n) /(p-1), u$ is a superminimizer in $\mathbf{B} \backslash\{0\}$ and hence $Q_{\alpha, p, n}$ is the best quasisubminimizer constant in $\mathbf{B} \backslash\{0\}$ for such $\alpha$. Similarly, for $\alpha \geq(p-n) /(p-1), u$ is a subminimizer in $\mathbf{B} \backslash\{0\}$, so $Q_{\alpha, p, n}$ must be the best quasisuperminimizer constant in $\mathbf{B} \backslash\{0\}$.

For $0<\alpha \leq 1-n / p, u$ is a superminimizer but not a quasiminimizer in $\mathbf{B} \backslash\{0\}$. Thus $u$ cannot be a quasisubminimizer there (and hence neither in $\mathbf{B}$ ).

Let us finally show that for $\alpha>1-n / p, u$ is a quasisubminimizer not only in $\mathbf{B} \backslash\{0\}$ but also in B. Clearly, $u \in W_{\text {loc }}^{1, p}(\mathbf{B})$. Let $\varphi \in \operatorname{Lip}_{c}(\mathbf{B})$ be nonpositive and $\widetilde{\varphi}=\max \{\varphi,-u\}$ so that $u+\widetilde{\varphi}=(u+\varphi)_{+}$and $|\nabla(u+\widetilde{\varphi})| \leq|\nabla(u+\varphi)|$. Then $\widetilde{\varphi} \in W_{0}^{1, p}(\mathbf{B} \backslash\{0\})$ and hence

$$
\int_{\varphi \neq 0}|\nabla u|^{p} d x \leq Q \int_{\varphi \neq 0}|\nabla(u+\widetilde{\varphi})|^{p} d x \leq Q \int_{\varphi \neq 0}|\nabla(u+\varphi)|^{p} d x,
$$

and thus $u$ is a $Q$-quasisubminimizer in $\mathbf{B}$ with the same quasisubminimizer constant $Q$ as in $\mathbf{B} \backslash\{0\}$.

\section{The case $p=n>1$}

Recall that we do not study $p=1$ at all in this paper.

\subsection{Powers.}

Theorem 7.1. Let $p=n>1$ and $u(x)=|x|^{\alpha}$. Then $u$ is a quasiminimizer in $\mathbf{B} \backslash\{0\}$ if and only if $\alpha=0$.

Proof. The case $\alpha=0$ is clear, so assume that $\alpha \neq 0$. Let $\varphi(r)=r^{\alpha}$.

As in the proof of Theorem 4.1 we have with $G=\left\{r: r_{1}<r<r_{2}\right\}$ and $R=$ $r_{2} / r_{1}>1$,

$$
\hat{I}_{\varphi}(G)=\frac{|\alpha|^{p}}{p \alpha-p+n} r_{1}^{p \alpha-p+n}\left(R^{p \alpha-p+n}-1\right)=\frac{|\alpha|^{n}}{n \alpha} r_{1}^{n \alpha}\left(R^{n \alpha}-1\right) .
$$

This time the minimizer of $\hat{I}$ is given by $\psi(r)=\log r$, and we have

$$
\hat{I}_{\psi}(G)=\int_{r_{1}}^{r_{2}}\left(\frac{1}{r}\right)^{p} r^{n-1} d r=\int_{r_{1}}^{r_{2}} \frac{d r}{r}=\log r_{2}-\log r_{1}=\log R .
$$

Let now $\eta=a \psi+b$, where we choose $a$ and $b$ so that $\eta=\varphi$ on $\partial G$, i.e. so that

$$
r_{1}^{\alpha}=a \log r_{1}+b \quad \text { and } \quad r_{2}^{\alpha}=a \log r_{2}+b .
$$

We only need to determine

Thus

$$
a=\frac{r_{2}^{\alpha}-r_{1}^{\alpha}}{\log r_{2}-\log r_{1}}=r_{1}^{\alpha} \frac{R^{\alpha}-1}{\log R} .
$$

$$
\hat{I}_{\eta}(G)=|a|^{n} \hat{I}_{\psi}(G)
$$

and we know that $\eta$ minimizes the $\hat{I}$-energy given the boundary values of $\varphi$.

Next we want to calculate, still letting $R=r_{2} / r_{1}$,

$$
\frac{\hat{I}_{\varphi}(G)}{\hat{I}_{\eta}(G)}=\frac{|\alpha|^{n}}{n \alpha} r_{1}^{n \alpha}\left(r_{1}^{\alpha} \frac{\left|R^{\alpha}-1\right|}{\log R}\right)^{-n} \frac{R^{n \alpha}-1}{\log R}=\frac{|\alpha|^{n}}{n \alpha} \frac{R^{n \alpha}-1}{\left|R^{\alpha}-1\right|^{n}}(\log R)^{n-1}=: k(R) .
$$


Both when $\alpha>0$ and when $\alpha<0$ it is easy to see that

$$
\lim _{R \rightarrow \infty} \frac{\left|R^{n \alpha}-1\right|}{\left|R^{\alpha}-1\right|^{n}}=1
$$

and thus that $\lim _{R \rightarrow \infty} k(R)=\infty$, as $n>1$, showing that $u$ is not a quasiminimizer in $\mathbf{B} \backslash\{0\}$.

Theorem 7.2. Let $p=n>1$ and $u(x)=|x|^{\alpha}$. Then $u$ is a $Q$-quasi(sub/super)minimizer and $Q$-quasi(sub/super)harmonic in $\mathbf{B}$ and $\mathbf{B} \backslash\{0\}$ as given in Table 3.

\begin{tabular}{|c|c|c|c|c|c|}
\hline $\begin{array}{c}p=n>1 \\
|x|^{\alpha}\end{array}$ & $\begin{array}{c}\text { quasi- } \\
\text { sub- } \\
\text { mini- } \\
\text { mizer } \\
\text { in } \mathbf{B} \backslash\{0\}\end{array}$ & $\begin{array}{l}\text { quasi- } \\
\text { sub- } \\
\text { mini- } \\
\text { mizer } \\
\text { in B }\end{array}$ & $\begin{array}{c}\text { quasi- } \\
\text { super- } \\
\text { mini- } \\
\text { mizer } \\
\text { in B }\end{array}$ & $\begin{array}{c}\text { quasi- } \\
\text { super- } \\
\text { mini- } \\
\text { mizer } \\
\text { in } \mathbf{B} \backslash\{0\}\end{array}$ & $\begin{array}{c}\text { quasi- } \\
\text { super- } \\
\text { harmo- } \\
\text { nic } \\
\text { in B }\end{array}$ \\
\hline$\alpha<0$ & $Q=1$ & Fails & Fails & Fails & Fails \\
\hline$\alpha=0$ & $Q=1$ & $Q=1$ & $Q=1$ & $Q=1$ & $Q=1$ \\
\hline$\alpha>0$ & $Q=1$ & $Q=1$ & Fails & Fails & Fails \\
\hline
\end{tabular}

Table 3.

Proof. The case $\alpha=0$ is clear. So assume that $\alpha \neq 0$.

By Theorem 3.1, $u$ is a subminimizer in $\mathbf{B} \backslash\{0\}$. Hence it follows from Theorem 7.1 that $u$ cannot be a quasisuperminimizer in $\mathbf{B} \backslash\{0\}$. Thus $u$ cannot be quasisuperharmonic in $\mathbf{B}$ nor a quasisuperminimizer in $\mathbf{B}$ either.

By Theorem 3.2, $u$ is a subminimizer in $\mathbf{B}$ when $\alpha \geq 0$. On the other hand, when $\alpha<0, u(0)=\infty$ and thus $u$ is not quasisubharmonic (nor a quasisubminimizer) in B.

\section{2. log-powers.}

Theorem 7.3. Let $p=n>1$ and $u(x)=(-\log |x|)^{\alpha}$. Then $u$ is a quasiminimizer in $\mathbf{B} \backslash\{0\}$ if and only if $\alpha>1-1 / n$ or $\alpha=0$.

Moreover, if $\alpha>1-1 / n$, then

$$
Q_{\alpha, n}=\frac{\alpha^{n}}{n \alpha-n+1}
$$

is the best quasiminimizer constant.

When $p=2$ (and $n=2$ ) one can easily see that $\alpha=Q \pm \sqrt{Q^{2}-Q}$. For $p=n>2, Q>1$ and $\alpha>1$, we have $\alpha<n \alpha-n+1<n \alpha$ and hence

$$
Q^{1 /(n-1)}<\alpha<(n Q)^{1 /(n-1)} .
$$

Proof. The case $\alpha=0$ is clear, so assume that $\alpha \neq 0$. Let $\varphi(r)=(-\log r)^{\alpha}$.

Let $G=\left\{r: r_{1}<r<r_{2}\right\}, 0<r_{1}<r_{2}<1, s_{1}=-\log r_{1}, s_{2}=-\log r_{2}<s_{1}$ and $S=s_{1} / s_{2}>1$. This time we get, assuming that $\alpha \neq 1-1 / n$,

$$
\begin{aligned}
\hat{I}_{\varphi}(G) & =\int_{r_{1}}^{r_{2}}|\alpha|^{n} \frac{(-\log r)^{n \alpha-n}}{r^{n}} r^{n-1} d r=\int_{r_{1}}^{r_{2}}|\alpha|^{n}(-\log r)^{n \alpha-n} \frac{d r}{r} \\
& =\frac{|\alpha|^{n}}{n \alpha-n+1}\left(s_{1}^{n \alpha-n+1}-s_{2}^{n \alpha-n+1}\right)=\frac{|\alpha|^{n}}{n \alpha-n+1} s_{2}^{n \alpha-n+1}\left(S^{n \alpha-n+1}-1\right) .
\end{aligned}
$$


The minimizer is given by $\psi(r)=-\log r$, and we have letting $\alpha=1$ above,

$$
\hat{I}_{\psi}(G)=s_{2}(S-1) \text {. }
$$

Let now $\eta=a \psi+b$, where we choose $a$ and $b$ so that $\eta=\varphi$ on $\partial G$, i.e. so that

$$
s_{1}^{\alpha}=a s_{1}+b \quad \text { and } \quad s_{2}^{\alpha}=a s_{2}+b .
$$

We only need to determine

$$
a=\frac{s_{1}^{\alpha}-s_{2}^{\alpha}}{s_{1}-s_{2}}=s_{2}^{\alpha-1} \frac{S^{\alpha}-1}{S-1} .
$$

Thus

$$
\hat{I}_{\eta}(G)=|a|^{n} \hat{I}_{\psi}(G),
$$

and we know that $\eta$ minimizes the $\hat{I}$-energy given the boundary values of $\varphi$.

Next we want to calculate

$$
\begin{aligned}
\frac{\hat{I}_{\varphi}(G)}{\hat{I}_{\eta}(G)} & =\frac{|\alpha|^{n}}{n \alpha-n+1} s_{2}^{n \alpha-n+1}\left(S^{n \alpha-n+1}-1\right) s_{2}^{n-n \alpha}\left(\frac{S-1}{\left|S^{\alpha}-1\right|}\right)^{n} \frac{1}{s_{2}(S-1)} \\
& =\frac{|\alpha|^{n}}{n \alpha-n+1} \frac{S^{n \alpha-n+1}-1}{S-1}\left(\frac{S-1}{\left|S^{\alpha}-1\right|}\right)^{n}=: k(S) .
\end{aligned}
$$

Case 1. $\alpha>1-1 / n$. In this case $n \alpha-n+1>0$ and $Q=\lim _{S \rightarrow \infty} k(S)=$ $\alpha^{n} /(n \alpha-n+1)$. Arguing as in the proof of Theorem 4.1 we see that $u$ is a $Q$ quasiminimizer and that $Q$ is the best quasiminimizer constant.

Case 2. $0<\alpha<1-1 / n$. In this case $n \alpha-n+1<0$ and thus $k(S)$ grows as $S^{n-1-n \alpha}$ for large $S$. As $n-1-n \alpha>0$ we see that $\lim _{S \rightarrow \infty} k(S)=\infty$, and thus $u$ is not a quasiminimizer in $\mathbf{B} \backslash\{0\}$.

Case 3. $\alpha<0$. In this case $k(S)$ grows as $S^{n-1}$ for large $S$ showing that $\lim _{S \rightarrow \infty} k(S)=\infty$, and thus $u$ is not a quasiminimizer in $\mathbf{B} \backslash\{0\}$. (This case can also be eliminated using the strong minimum principle as in the case $\alpha>0$ in the proof of Theorem 6.2.)

Case 4. $\alpha=1-1 / n$. In this case

$$
\begin{aligned}
\hat{I}_{\varphi}(G) & =\int_{r_{1}}^{r_{2}}|\alpha|^{n}(-\log r)^{n \alpha-n} \frac{d r}{r}=\int_{r_{1}}^{r_{2}}|\alpha|^{n} \frac{d r}{-r \log r} \\
& =\alpha^{n}\left(\log s_{1}-\log s_{2}\right)=\alpha^{n} \log S .
\end{aligned}
$$

Thus

$$
\frac{\hat{I}_{\varphi}(G)}{\hat{I}_{\eta}(G)}=\frac{\alpha^{n} \log S}{s_{2}(S-1)} s_{2}^{n-n \alpha}\left(\frac{S-1}{S^{\alpha}-1}\right)^{n}=\frac{\alpha^{n} \log S}{S-1}\left(\frac{S-1}{S^{\alpha}-1}\right)^{n}=: k(S) .
$$

It is easy to see that $k(S)$ grows as $\log S$, as $S \rightarrow \infty$, and thus that $\lim _{S \rightarrow \infty} k(S)=\infty$, and hence $u$ is not a quasiminimizer in $\mathbf{B} \backslash\{0\}$.

Theorem 7.4. Let $p=n>1, u(x)=(-\log |x|)^{\alpha}$ and let $Q_{\alpha, n}$ be as in (7.1). Then $u$ is a $Q$-quasi(sub/super)minimizer and $Q$-quasi(sub/super)harmonic in $\mathbf{B}$ and $\mathbf{B} \backslash\{0\}$ as given in Table 4. Moreover, $Q$ in Table 4 is the best quasi(sub/super)minimizer constant. 


\begin{tabular}{|c|c|c|c|c|c|}
\hline $\begin{array}{l}p=n>1 \\
(-\log |x|)^{\alpha}\end{array}$ & $\begin{array}{c}\text { quasi- } \\
\text { sub- } \\
\text { mini- } \\
\text { mizer } \\
\text { in } \mathbf{B} \backslash\{0\}\end{array}$ & $\begin{array}{l}\text { quasi- } \\
\text { sub- } \\
\text { mini- } \\
\text { mizer } \\
\text { in B }\end{array}$ & $\begin{array}{c}\text { quasi- } \\
\text { super- } \\
\text { mini- } \\
\text { mizer } \\
\text { in B }\end{array}$ & $\begin{array}{c}\text { quasi- } \\
\text { super- } \\
\text { mini- } \\
\text { mizer } \\
\text { in } \mathbf{B} \backslash\{0\}\end{array}$ & $\begin{array}{c}\text { quasi- } \\
\text { super- } \\
\text { harmo- } \\
\text { nic } \\
\text { in B }\end{array}$ \\
\hline$\overline{\alpha<<0}$ & $Q=1$ & $\overline{Q Q=1}$ & Fails & Fails & Fails \\
\hline$\alpha=0$ & $Q=1$ & $Q=1$ & $Q=1$ & $Q=1$ & $Q=1$ \\
\hline $0<\alpha<1-\frac{1}{n}$ & Fails & Fails & $Q=1$ & $Q=1$ & $Q=1$ \\
\hline$\alpha=1-\frac{1}{n}$ & Fails & Fails & Fails & $Q=1$ & $Q=1$ \\
\hline $1-\frac{1}{n}<\alpha<1$ & $Q=Q_{\alpha, n}$ & Fails & Fails & $Q=1$ & $Q=1$ \\
\hline$\alpha=1$ & $Q=1$ & Fails & Fails & $Q=1$ & $Q=1$ \\
\hline$\alpha>1$ & $Q=1$ & Fails & Fails & $Q=Q_{\alpha, n}$ & $Q=Q_{\alpha, n}$ \\
\hline
\end{tabular}

Table 4.

Remark 7.5. Recall that, by Remark $2.4,(-\log |x|)^{\alpha}$ is a $Q$-quasisubminimizer in $\mathbf{B} \backslash\{0\}$ or $\mathbf{B}$ if and only if it is Q-quasisubharmonic there, and $u$ is a $Q$ quasisuperminimizer in $\mathbf{B} \backslash\{0\}$ if and only if it is $Q$-quasisuperharmonic there.

Recall that Remark 2.4 also shows that the last two columns are the same in this case.

Proof. The case $\alpha=0$ is clear. Moreover, the last two columns are the same by Remark 2.4.

Let us first note that, $u \in W_{\text {loc }}^{1, p}(\mathbf{B})$ if and only if $\alpha<1-1 / n$, showing that for $\alpha \geq 1-1 / n, u$ is neither a quasisubminimizer nor a quasisuperminimizer in $\mathbf{B}$.

Case 1. $\alpha<0$. In this case, $u$ is a subminimizer in $\mathbf{B}$ (and thus in $\mathbf{B} \backslash\{0\}$ ), by Theorem 3.4. As it is not a quasiminimizer in $\mathbf{B} \backslash\{0\}$, by Theorem 7.3, it cannot be a quasisuperminimizer there (and thus not in $\mathbf{B}$ either).

Case 2. $0<\alpha \leq 1$. In this case, $u$ is superharmonic in $\mathbf{B}$ by Theorem 3.4. If $0<\alpha \leq 1-1 / n$, then $u$ is not a quasiminimizer in $\mathbf{B} \backslash\{0\}$, by Theorem 7.3, and hence it cannot be a quasisubminimizer there (and thus not in $\mathbf{B}$ either). By Theorem 3.4, $u$ is a superminimizer in $\mathbf{B}$ for $0<\alpha<1-1 / n$.

If $1-1 / n<\alpha \leq 1$, then $u$ is a quasiminimizer in $\mathbf{B} \backslash\{0\}$, by Theorem 7.3 , with $Q_{\alpha, n}$ being the best quasiminimizer constant. As it is a superminimizer in $\mathbf{B} \backslash\{0\}, Q_{\alpha, n}$ must be the best quasisubminimizer constant for $u$ in $\mathbf{B} \backslash\{0\}$. Note that $Q_{1, n}=1$.

Case 3. $\alpha>1$. Then $u$ is a quasiminimizer in $\mathbf{B} \backslash\{0\}$, by Theorem 7.3, with $Q_{\alpha, n}$ being the best quasiminimizer constant. As it is a subminimizer in $\mathbf{B} \backslash\{0\}$, by Theorem 3.3, $Q_{\alpha, n}$ must be the best quasisuperminimizer constant for $u$ in $\mathbf{B} \backslash\{0\}$.

\section{Local quasiminimizers}

In $\mathbf{R}^{n}$ it is well known that $p$-harmonicity is a local property, i.e. if a function is $p$-harmonic in $\Omega_{1}$ and $\Omega_{2}$ then it is $p$-harmonic in $\Omega_{1} \cup \Omega_{2}$. We shall see in this section that this so called sheaf property fails for quasiminimizers. 
Let us make the following definition.

Definition 8.1. We say that $u$ is a local $Q$-quasiminimizer in $\Omega$ if we can find finitely or countably many open sets $\Omega_{1}, \Omega_{2}, \ldots$, such that $\Omega=\bigcup_{j} \Omega_{j}$ and such that $u$ is a $Q$-quasiminimizer in $\Omega_{j}$ for all $j$.

Proposition 8.2. Let $p>1, n \geq 1, \alpha \in \mathbf{R}, u(x)=|x|^{\alpha}$ and $\varepsilon>0$. Then $u$ is a local $(1+\varepsilon)$-quasiminimizer in $\mathbf{R}^{n} \backslash\{0\}$.

Proof. This is clear if $\alpha=0$. By the proof of Theorem 4.1 we see that $k$ therein satisfies $\lim _{R \rightarrow 1} k(R)=1$ (if $\alpha=1-n / p$, we use Proposition 4.2). Hence we can find $\tau>1$ such that $\sup _{1 \leq R \leq \tau^{2}} k(R) \leq 1+\varepsilon$. Let now $\Omega_{j}=\left\{x \in \mathbf{R}^{n}: \tau^{j}<|x|<\tau^{j+2}\right\}$, $j \in \mathbf{Z}$. With the notation as in the proof of Theorem 4.1, we see that $1<R=$ $r_{2} / r_{1}<\tau^{2}$ in $\Omega_{j}$ and it follows that $u$ is a $(1+\varepsilon)$-quasiminimizer in $\Omega_{j}, j \in \mathbf{Z}$.

Together with our earlier results, Proposition 8.2 gives plenty of examples of local $(1+\varepsilon)$-quasiminimizers which are not quasiminimizers. There is a similar result for $p=n>1$ and log-powers.

It was pointed out by Kinnunen-Martio [15] that being a quasiminimizer is not a local property. Judin [14], Example 4.2.4, gave an explicit example of a local quasiminimizer on $(0, \infty)$, for $p=2$, which is not a quasiminimizer on $(0, \infty)$.

On the other hand, we have the following result in the opposite direction.

Proposition 8.3. Let $Q>1$. There is a $Q$-quasiminimizer which is not a local $Q^{\prime}$-quasiminimizer for any $Q^{\prime}<Q$.

Proof. Let $n=1$ and $p=2$. Then we can find $a>0$ such that $Q=(a+1)^{2} / 4 a$. Let also $1<Q^{\prime}<Q$. In this case,

$$
u(x)= \begin{cases}x, & x \leq 0, \\ a x, & x \geq 0\end{cases}
$$

is a $Q$-quasiminimizer in $\mathbf{B}=(-1,1) \subset \mathbf{R}$, but not a $Q^{\prime}$-quasiminimizer in $B(0, r)$ for any $0<r<1$, which is seen by a straightforward calculation and was obtained by Judin [14], Example 4.0.25. The example of Judin was extended to arbitrary $p>1$ by Uppman [24], Section 2.2.3. It follows that $u$ is not a $Q^{\prime}$-quasiminimizer in any neighbourhood of 0 , and hence not a local $Q^{\prime}$-quasiminimizer in $\mathbf{B}$.

Proposition 8.4. Let $a, b \in \mathbf{R}, a<b$, and let $u \in C^{1}([a, b])$ be such that

$$
u^{\prime}(x) \neq 0 \quad \text { for } x \in[a, b] \text {. }
$$

Let further

$$
Q=\left(\frac{\max _{x \in[a, b]}\left|u^{\prime}(x)\right|}{\min _{x \in[a, b]}\left|u^{\prime}(x)\right|}\right)^{p} .
$$

Then $u$ is a $Q$-quasiminimizer in $(a, b)$.

Corollary 8.5. Let $a, b \in \mathbf{R}, a<b$, and let $u \in C^{1}((a, b))$ be such that $u^{\prime}(x) \neq 0$ for $a<x<b$. Then $u$ is a local $(1+\varepsilon)$-quasiminimizer in $(a, b)$ for every $\varepsilon>0$.

In fact the proof here is valid also with smooth weights, and can thus be applied for quasiminimizers with respect to the energy $\hat{I}$. This and the arguments at the end of the proof of Theorem 4.1 can be used to give an alternative proof of Proposition 8.2.

Let us also mention that Martio-Sbordone [21] studied quasiminimizers on $\mathbf{R}$ quite extensively. 
Proof of Proposition 8.4. We may assume that $u$ is strictly increasing. Let

$$
M=\max _{x \in[a, b]}\left|u^{\prime}(x)\right|, \quad m=\min _{x \in[a, b]}\left|u^{\prime}(x)\right|,
$$

and $a<c<d<b$. Let further $v$ be the minimizer in $(c, d)$ with $u$ as boundary values, i.e. $v$ is linear. Then $m \leq v^{\prime} \leq M$ in $(c, d)$. So

$$
\int_{c}^{d}\left|u^{\prime}\right|^{p} d x \leq(d-c) M^{p} \leq Q(d-c) m^{p} \leq Q \int_{c}^{d}\left|v^{\prime}\right|^{p} d x
$$

Hence $u$ is a $Q$-quasiminimizer in $(a, b)$.

Proof of Corollary 8.5. Let $\varepsilon>0$. For each $x \in(a, b)$ we can find $y_{x} \in(x, b)$ such that

$$
\left(\frac{\max _{t \in\left[x, y_{x}\right]}\left|u^{\prime}(t)\right|}{\min _{t \in\left[x, y_{x}\right]}\left|u^{\prime}(t)\right|}\right)^{p}<1+\varepsilon .
$$

We can next find a countable subcover $\left\{I_{j}\right\}_{j=1}^{\infty}$ of $\left\{\left(x, y_{x}\right)\right\}_{x \in(a, b)}$, where $I_{j}=\left(x_{j}, y_{x_{j}}\right)$. Thus $(a, b)=\bigcup_{j=1}^{\infty} I_{j}$. By Proposition 8.4, $u$ is a $(1+\varepsilon)$-quasiminimizer in $I_{j}$ for each $j$. Hence $u$ is a local $(1+\varepsilon)$-quasiminimizer in $(a, b)$.

\section{References}

[1] BJöRn, A.: A weak Kellogg property for quasiminimizers. - Comment. Math. Helv. 81, 2006, 809-825.

[2] BJöRN, A.: Removable singularities for bounded $p$-harmonic and quasi(super)harmonic functions on metric spaces. - Ann. Acad. Sci. Fenn. Math. 31, 2006, 71-95.

[3] BJÖRN, A.: A regularity classification of boundary points for $p$-harmonic functions and quasiminimizers. - J. Math. Anal. Appl. 338, 2008, 39-47.

[4] BJÖRN, A.: Cluster sets for Sobolev functions and quasiminimizers. - J. Anal. Math. (to appear).

[5] BJörn, A., J. BJörn, and N. Marola: BMO, local integrability, Harnack and Caccioppoli inequalities for quasisuperharmonic functions. - Ann. Inst. H. Poincaré Anal. Non Linéaire 27, 2010, 1489-1505.

[6] BJörn, A., and N. MAROla: Moser iteration for (quasi)minimizers on metric spaces. Manuscripta Math. 121, 2006, 339-366.

[7] BJÖRn, A., and O. MARTio: Pasting lemmas and characterizations of boundary regularity for quasiminimizers. - Results Math. 55, 2009, 265-279.

[8] BJÖRN, J.: Boundary continuity for quasiminimizers on metric spaces. - Illinois J. Math. 46, 2002, 383-403.

[9] BJÖRN, J.: Necessity of a Wiener type condition for boundary regularity of quasiminimizers and nonlinear elliptic equations. - Calc. Var. Partial Differential Equations 35, 2009, 481-496.

[10] DiBenedetto, E., and N. S. Trudinger: Harnack inequalities for quasiminima of variational integrals. - Ann. Inst. H. Poincaré Anal. Non Linéaire 1, 1984, 295-308.

[11] Giaquinta, M., and E. Giusti: On the regularity of the minima of variational integrals. Acta Math. 148, 1982, 31-46.

[12] Giaquinta, M. and E. Giusti: Quasi-minima. - Ann. Inst. H. Poincaré Anal. Non Linéaire 1, 1984, 79-107.

[13] Heinonen, J., T. Kilpeläınen, and O. Martio: Nonlinear potential theory of degenerate elliptic equations. - 2nd ed., Dover, Mineola, NY, 2006. 
[14] Judin, P.T.: Onedimensional quasiminimizers and quasisuperminimizers [Yksiulotteiset kvasiminimoijat ja kvasisuperminimoijat]. - Licentiate thesis, Department of Mathematics and Statistics, University of Helsinki, Helsinki, 2006 (in Finnish).

[15] Kinnunen, J., and O. Martio: Potential theory of quasiminimizers. - Ann. Acad. Sci. Fenn. Math. 28, 2003, 459-490.

[16] Kinnunen, J., and N. Shanmugalingam: Regularity of quasi-minimizers on metric spaces. - Manuscripta Math. 105, 2001, 401-423.

[17] Martio, O.: Boundary behavior of quasiminimizers and Dirichlet finite PWB solutions in the borderline case. - Report in Math. 440, Department of Mathematics and Statistics, University of Helsinki, Helsinki, 2006.

[18] Martio, O.: Reflection principle for quasiminimizers. - Funct. Approx. Comment. Math. 40, 2009, 165-173.

[19] Martio, O.: Quasiminimizers - definitions, constructions and capacity estimates. - Lectures held at the conference "Nonlinear problems for $\Delta_{p}$ and $\Delta$ ", Linköping University, Linköping, 2009, http://www.mai.liu.se/TM/conf09/martio.pdf.

[20] Martio, O.: Capacity and potential estimates for quasiminimizers. - Complex Anal. Oper. Theory (to appear), doi:10.1007/s11785-010-0074-5.

[21] Martio, O., and C. Sbordone: Quasiminimizers in one dimension: integrability of the derivative, inverse function and obstacle problems. - Ann. Mat. Pura Appl. 186, 2007, 579-590.

[22] Tolksdorf, P.: Remarks on quasi(sub)minima. - Nonlinear Anal. 10, 1986, 115-120.

[23] Trudinger, N.S.: On Harnack type inequalities and their application to quasilinear elliptic equations. - Comm. Pure Appl. Math. 20, 1967, 721-747.

[24] Uppman, H.: The reflection principle for one-dimensional quasiminimizers. - Master's thesis, Linköping University, Linköping, 2009, http://urn.kb.se/resolve?urn=urn:nbn:se:liu:diva19162.

[25] Ziemer, W. P.: Boundary regularity for quasiminima. - Arch. Rational Mech. Anal. 92, 1986, 371-382.

Received 10 May 2010 